\title{
Serendipitous VLBI detection of rapid, large-amplitude, intraday variability in QSO 1156+295
}

\author{
T. Savolainen ${ }^{1}$ and Y. Y. Kovalev ${ }^{1,2}$ \\ 1 Max-Planck-Institut für Radioastronomie, Auf dem Hügel 69, 53121 Bonn, Germany \\ e-mail: [tsavolainen; ykovalev] @mpifr-bonn.mpg.de \\ 2 Astro Space Center of Lebedev Physical Institute, Profsoyuznaya 84/32, 117997 Moscow, Russia \\ Received 19 June 2008 / Accepted 18 August 2008

\section{ABSTRACT}

\begin{abstract}
Aims. We report a serendipitous detection of rapid, large amplitude flux density variations in the highly core-dominated, flat-spectrum radio quasar 1156+295 during an observing session at the Very Long Baseline Array (VLBA).

Methods. The source was observed as a part of the MOJAVE survey programme with the VLBA at $15 \mathrm{GHz}$ on February 5, 2007. Large amplitude variability in the correlated flux density, unexplainable in terms of the source structure, was first discovered while processing the data, and later confirmed by calibrating the antenna gains using 24 other sources observed in the experiment. Results. The source shows variations in the correlated flux density as high as $40 \%$ on a timescale of only $2.7 \mathrm{~h}$. This places $1156+295$ between the classical IDV sources and the so-called intra-hour variables. The observed variability timescale and the modulation index of $13 \%$ are consistent with interstellar scintillation by a nearby, highly turbulent scattering screen. The large modulation index at $15 \mathrm{GHz}$ implies a scattering measure that is atypically high for a high galactic latitude source such as $1156+295$.
\end{abstract}

Key words. galaxies: active - galaxies: jets - galaxies: quasars: individual: QSO 1156+295 - techniques: interferometric scattering - ISM: structure

\section{Introduction}

Intraday variability (IDV) of compact, radio-loud AGN at cmwavelengths was first discovered in the mid-1980s (Witzel et al. 1986; Heeschen et al. 1987) and both source-intrinsic and extrinsic mechanisms for IDV have been vigorously studied (for a review, see e.g. Wagner \& Witzel 1995). Detection of time delays in the variability pattern arrival times between widely separated telescopes, as well as observed annual modulation of the variability timescale, have shown conclusively that interstellar scintillation (ISS) is the cause of intra-hour flux density variations observed in the three most extreme IDV sources PKS 0405-385 (Jauncey et al. 2000), J1819+3845 (Dennett-Thorpe \& de Bruyn 2002, 2003), and PKS 1257-326 (Bignall et al. 2003, 2006). Evidence for the ISS origin of IDV was found also in the cases of 0917+624 (Rickett et al. 2001; Jauncey \& Macquart 2001; Fuhrmann et al. 2002), PKS 1519-273 (Jauncey et al. 2003), and J1128+5925 (Gabányi et al. 2007). This provided the possibility to study both small-scale spatial fluctuations in the interstellar medium and the structure of compact radio sources on the microarcsecond scale. Unfortunately, the extreme IDV sources, which are the most suitable for these studies, are rare. By "extreme", we mean sources showing variability on timescales of a few hours or less and with an rms amplitude of modulation of over $10 \%$. In this Letter, we report the serendipitous discovery of very large amplitude IDV in quasar $1156+295$ with a timescale of variations shorter than $3 \mathrm{~h}$. This discovery is also unusual because it was achieved by a VLBI experiment.

$1156+295(4 \mathrm{C}+29.45)$ is an optically violently variable quasar at $z=0.729$ (Burbidge 1968), which has Galactic coordinates $l=199.4^{\circ}, b=+78.4^{\circ}$. The source is strongly variable throughout the electromagnetic spectrum from radio to $\gamma$-rays. At radio frequencies, $1156+295$ shows significant long-term

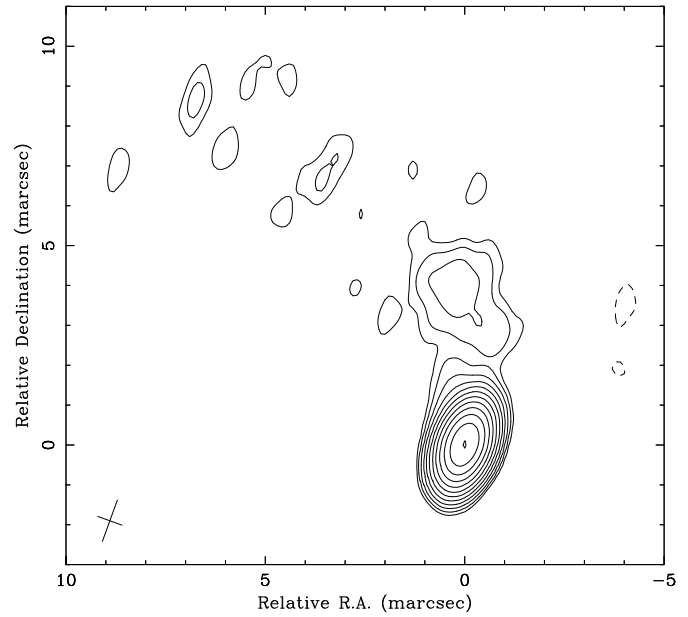

Fig. 1. Naturally weighted $15 \mathrm{GHz}$ VLBA image of $1156+295$ observed on February 5, 2007. The map peak brightness is $1.46 \mathrm{Jy}^{\text {beam }}{ }^{-1}$. The contours begin at $0.7 \mathrm{mJy}_{\text {beam }}^{-1}$ and increase in steps of 2 . Since the source flux density varied significantly during the observation, an amplitude self-calibration had to be used early in the imaging process. This may affect the image quality.

variability on a timescale of months (e.g. Kovalev et al. 2002). Wills et al. (1983) reported a range of at least 5 mag in the optical brightness, and in gamma-rays Sreekumar et al. (1996) detected an order of magnitude variability using the EGRET instrument onboard the Compton Gamma Ray Observatory. During high radio brightness states, $1156+295$ appears to be highly core-dominated in VLBI images (95\% at some epochs, Kovalev et al. 2005; see also Fig. 1). Superluminal motion at speeds 


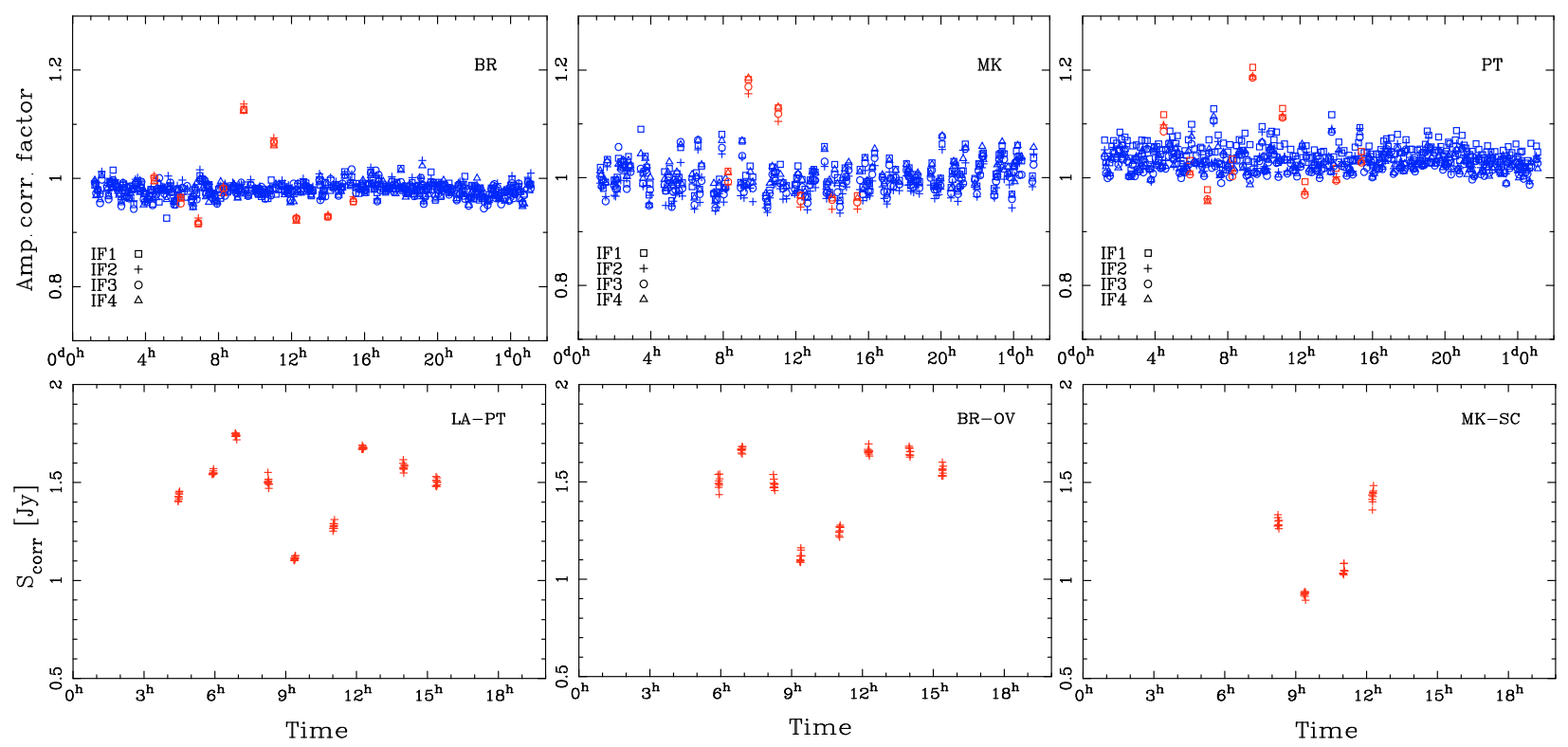

Fig. 2. Top: amplitude self-calibration solutions of three example antennas (Brewster, Mauna Kea, and Pie Town) for the VLBA experiment on February 5, 2007. There is one solution per scan, and IFs are kept separate. Low elevation scans $\left(<15^{\circ}\right)$ are excluded from the figure. Red symbols correspond to scans of 1156+295 and blue symbols correspond to all other sources. The obvious discrepancy between the solutions for $1156+295$ and the other sources is present at all ten antennas. Bottom: examples of the correlated flux density curves of $1156+295$ at three baselines after calibration of antenna gains by amplitude correction factors interpolated from the nearby scans of other sources. Time is in UT.

ranging from $3.5 h^{-1} c$ to $14.1 h^{-1} c$ were reported by e.g. Piner \& Kingham (1997), Jorstad et al. (2001), Hong et al. (2004), and Kellermann et al. (2004).

In the optical bands, $1156+295$ shows rapid variations on timescales of between less than 30 min and 3 days (Wills et al. 1983; Raiteri et al. 1998). The first IDV detection of $1156+295$ at radio frequencies was reported by Lovell et al. (2003), who observed the source with the VLA at $5 \mathrm{GHz}$ in January 2002. Their study measured rms flux density variations of $167 \mathrm{mJy}$ during 3 nights of observations and a variability timescale of $\sim 24 \mathrm{~h}$. The relative amplitude of variations observed by Lovell et al. (2003) was far smaller than reported here.

Throughout this paper, we use the following cosmological parameters: $H_{0}=73 \mathrm{~km} \mathrm{~s}^{-1} \mathrm{Mpc}^{-1}, \Omega_{\mathrm{M}}=0.27$, and $\Omega_{\Lambda}=0.73$. The angular scaling conversion for $z=0.729$ is $7.07 \mathrm{pc} \mathrm{mas}^{-1}$.

\section{Observations and analysis}

Quasar 1156+295 and 24 other compact radio sources were observed with the NRAO's Very Long Baseline Array (VLBA) at $15 \mathrm{GHz}$ as a part of the MOJAVE project (Lister \& Homan 2005) on February 5, 2007 ${ }^{1}$. The entire observing session had a duration of $24 \mathrm{~h}$, which included 9 scans of $1156+295$, each lasting $4.7 \mathrm{~min}$. The signal was recorded in dual-polarisation mode: 4 IFs (each with $8 \mathrm{MHz}$ bandwidth) per circular polarisation and 1-bit sampling. The data from the experiment were calibrated following standard procedures of VLBI data reduction (see e.g. Lister \& Homan 2005). After a priori amplitude calibration and fringe fitting, the correlated flux density of 1156+295 showed significant, correlated, temporal variability from scan to scan on every baseline: between $7 \mathrm{~h}$ and $12 \mathrm{~h}$ UT, it first dropped by about $0.6 \mathrm{Jy}$ and then increased again. The dip can be seen on every baseline, which excludes the source structure as the cause

\footnotetext{
1 We note that $1156+295$ was observed several times in the MOJAVE survey and its IDV behaviour during the rest of the MOJAVE epochs will be analysed in Kuchibhotla et al. (in prep.).
}

of the variations. The system temperature measurements used in the a priori amplitude calibration do not show any jumps that could explain the dip.

To ascertain whether the variability is genuine or due to amplitude calibration problems, we first imaged and self-calibrated the $(u, v)$ data of $1156+295$ in a normal manner and then compared the resulting antenna gain correction factors with those derived from the self-calibration of the other 24 sources observed in the same session. Amplitude self-calibration with a 5-min solution interval was indeed able to remove the variability of $1156+295$, but it produced gain amplitude correction factors that were - for every antenna - significantly offset from the gain corrections determined from the 24 other sources in the experiment (see examples in Fig. 2). This can be explained if the variability is genuine but does not alter the shape of the source's visibility function significantly during the experiment, e.g. if the variability occurs in one dominant compact component. Since the antenna gain is not source-dependent, calibration errors cannot account for the above-described behaviour.

Although improbable, there is a possibility that the observed variations could be due to a source-specific correlator problem. In such a case, however, one would expect to see discrepant solutions from the fringe fitting. We carefully checked the fringe-fit solutions and could not find any anomalies. Therefore, an instrumental effect that originates in the correlator seems highly unlikely. Taken together, we conclude from the above arguments that the variations in the correlated flux density of $1156+295$ are due to genuine intraday variability observed in the source.

To study rigorously the observed IDV event in $1156+295$, the residual errors in the antenna gains, which remain after the a priori amplitude calibration, need to be corrected. Since a large number of sources were observed during the VLBA session with their scans interleaved, it was possible to estimate the antenna gain correction factors for the scans of $1156+295$ by linearly interpolating the amplitude self-calibration solutions from the scans of the other sources that were observed within one hour of each scan of $1156+295$. Before interpolation, the scans at 


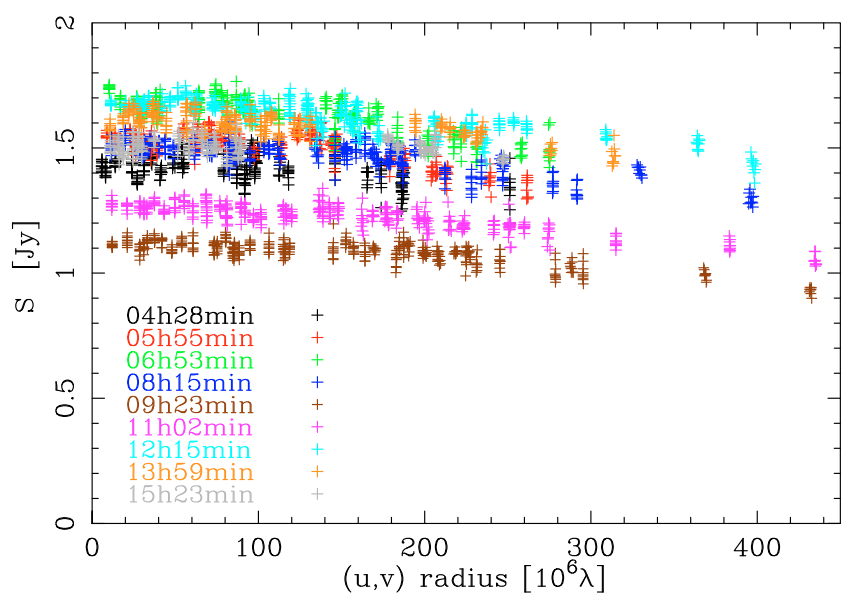

Fig. 3. Calibrated correlated Stokes I flux density of 1156+295 (in Jy) as a function of $(u, v)$ radius (in $\mathrm{M} \lambda)$ for each individual scan of the VLBI experiment on February 5, 2007. The data has been averaged over the IFs and for $30 \mathrm{~s}$ in time. The different scans are shown in different colours with the scan start time in UT indicated in the legend.

elevations below $15^{\circ}$ were removed. The bottom panels in Fig. 2 show examples of correlated flux density curves on three baselines that were calibrated in this way. The variations in the correlated flux density are very similar at short (LA-PT), intermediate (BR-OV), and long (MK-SC) baselines. Again, this is expected, if the variability occurs in a single dominant compact component. In Fig. 3, we plot the calibrated correlated flux density of $1156+295$ as a function of $(u, v)$ radius for each individual scan. The figure shows that the shape of the visibility function, $\mathcal{V}\left(r_{u, v}\right)$, changes little from scan to scan, although the correlated flux density changes by $0.6 \mathrm{Jy}$. To analyse possible small changes in the shape of $\mathcal{V}\left(r_{u, v}\right)$, we constructed the mean visibility function of the observation, $\left\langle\mathcal{V}\left(r_{u, v}\right)\right\rangle$, by averaging the normalised visibilities of all individual scans. The correlated flux density of each scan was first binned into $50 \mathrm{M} \lambda$ wide bins in projected $(u, v)$ spacing and then normalised to 1.0 at $r_{u, v}=25 \mathrm{M} \lambda$. We subtracted $\left\langle\mathcal{V}\left(r_{u, v}\right)\right\rangle$ from the binned $\mathcal{V}\left(r_{u, v}\right)$ of each individual scan and inspected the residuals. Some variability was found: $\mathcal{V}\left(r_{u, v}\right)$ at $200 \lesssim r_{u, v} \lesssim 300 \mathrm{M} \lambda$ increased by $8 \%$ during the observation, which is more than the $\approx 3 \%$ variability expected from the time variable response of the interferometer to the faint jet. However, the variability turned out to be solely due to the baselines to St. Croix, and therefore probably was due to calibration inaccuracies of the antenna. Consequently, we are unable to confirm conclusively the detection of time variability in $\mathcal{V}\left(r_{u, v}\right)$. An upper limit to variability is $8 \%$.

We constructed an integrated VLBA flux density curve for $1156+295$ by averaging the correlated flux density at projected baselines between 6-100 M $\lambda$ (Fig. 4). Since $\mathcal{V}\left(r_{u, v}\right)$ was essentially flat in this range (Fig. 3), the averaging provided a good estimate of the integrated emission coming from angular scales $\$ 30$ mas. Averaging also reduces the errors due to inaccurate amplitude calibration, because these are antenna-specific.

The two important quantities in the analysis of IDV are the modulation index and the characteristic timescale of variability. We consider the average of the peak-to-trough and troughto-peak times of the large dip to be the timescale of variability, which is therefore $t_{\mathrm{var}}=2.7 \pm 0.5 \mathrm{~h}$. We note that our $t_{\mathrm{var}}$ is approximately a factor of 1.7 times longer than the timescale on which the autocorrelation function of intensity fluctuations reaches a fraction $1 / e$ of its maximum value (Jauncey $\&$ Macquart 2001). The modulation index $m$, defined to be the

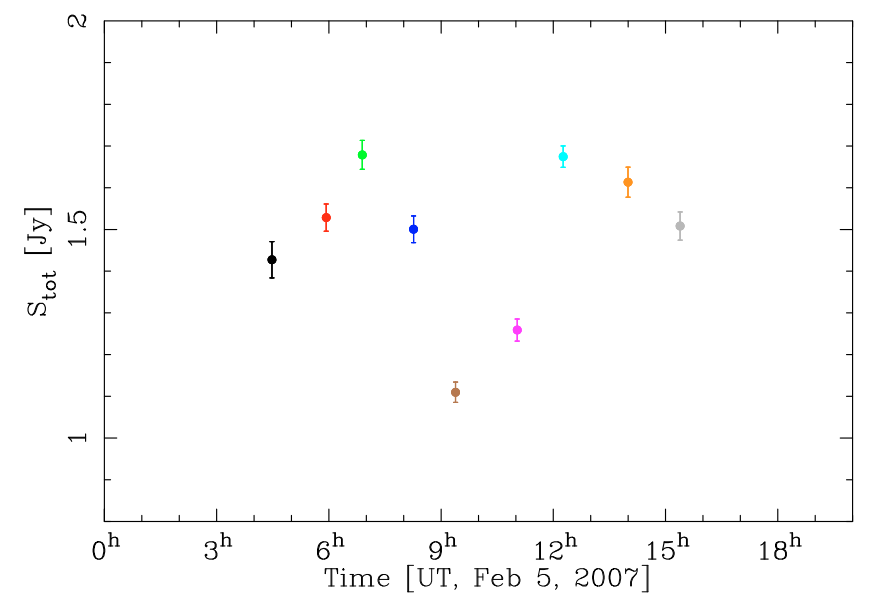

Fig. 4. Integrated flux density curve of $1156+295$, obtained by averaging the correlated flux density at projected baselines shorter than $100 \mathrm{M} \lambda$. The error bars represent the standard deviation of the correlated flux density (at baselines $<100 \mathrm{M} \lambda$ ) in each scan. Colour coding of the scans is the same as in Fig. 3.

standard deviation of the source flux density divided by the mean source flux density, is $13 \pm 3 \%$ for the flux density curve in Fig. 4 . The uncertainties in $t_{\mathrm{var}}$ and $m$ were estimated following the analysis in Dennett-Thorpe \& de Bruyn (2003). The modulation index is much higher than the values typically observed for IDV sources at frequencies above $5 \mathrm{GHz}$ (Kedziora-Chudczer et al. 2001; Kraus et al. 2003). For $1156+295$, Lovell et al. (2003) reported $m=5.8 \%$ at $5 \mathrm{GHz}$ in 2002 .

\section{Discussion}

If we assumed a source-intrinsic origin of the observed variability in $1156+295$, it would imply, by light travel time arguments, a brightness temperature of $\gtrsim 2 \times 10^{19} \mathrm{~K}$, which is far in excess of the inverse Compton (IC) limit of $10^{12} \mathrm{~K}$ (Kellermann \& Pauliny-Toth 1969). A Doppler factor higher than $\sim 270$ would be then required to avoid the IC catastrophe. This would cause severe problems with the energy requirements in the source (Begelman et al. 1994). Extremely fast jets are also unlikely in the light of VLBI monitoring surveys, which indicate that the maximum jet Lorentz factor $\Gamma$ is $30-40$ (Cohen et al. 2007). The standard model of incoherent synchrotron radiation from relativistic electrons in the jet would therefore have significant difficulties in explaining the observed variability, if it were intrinsic. For this reason, we concentrate on two possible extrinsic causes: interstellar scintillation and an extreme scattering event.

Propagation effects in the ionised medium of the Milky Way will cause a sufficiently compact source to scintillate (for review of ISS, see e.g. Rickett 1990; Goodman 1997). Since the observed $m$ in $1156+295$ is clearly below $100 \%$, it is reasonable to assume that diffractive scintillation is fully quenched and to consider only refractive ISS. The observed $t_{\mathrm{var}}$ and $m$ constrain the properties of a possible scattering screen and the size of the scintillating source. Because of the high $m$ in our case, it appears likely that our observing frequency is close to the critical frequency $v_{\mathrm{s}}$ between strong and weak scattering regimes. Goodman (1997) provided a formula for $m$ that can be used to interpolate solutions close to $v_{\mathrm{s}}$, where the asymptotic solutions for strong and weak regimes break down. Combining his Eqs. (10), (12), (18), (19), and (20), and assuming a Gaussian brightness profile for the source, we calculated the screen distance, $D_{\mathrm{scr}}$, and the $F W H M$ size of the scintillating source $\theta_{\mathrm{s}}^{F W H M}$ 

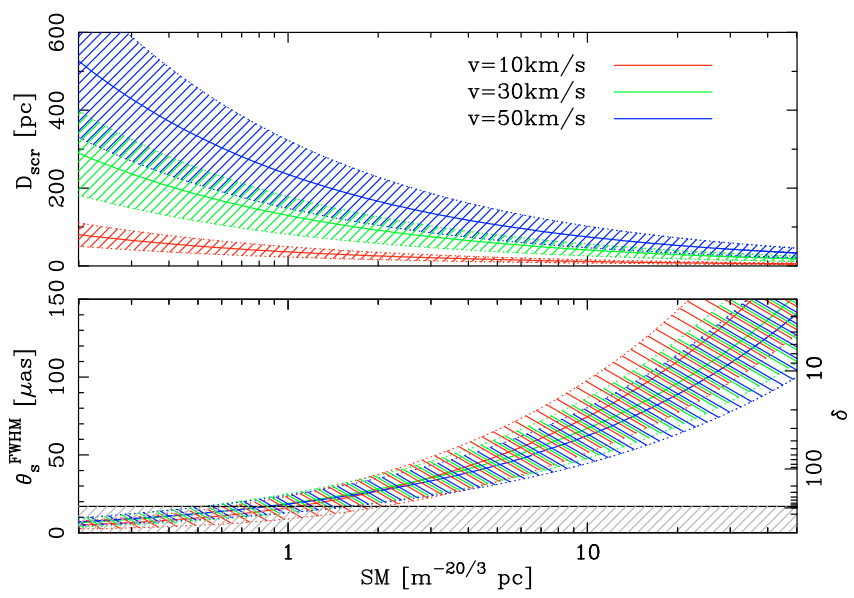

Fig. 5. Parameters of the possible scattering screen. Top: the distance to the screen as a function of the scattering measure $S M$. Different screen velocities are shown in different colours. Bottom: the scintillating source size as a function of $S M$. The right edge of the panel gives the Doppler factor if $\theta_{\mathrm{s}}^{F W H M}$ is equal to equipartition size. The grey shaded area corresponds to $\theta_{\mathrm{s}}^{F W H M}<17 \mu \mathrm{as}$. In both panels, the hatched region around the solid lines gives $1 \sigma$ error for the plotted quantities. The $+1 \sigma$ lines in the $\theta_{\mathrm{s}}^{F W H M}$ plot roughly correspond to $-1 \sigma$ lines in the $D_{\text {scr }}$ plot and vice versa.

as a function of scattering measure $S M=\int_{0}^{d} C_{N}^{2} \mathrm{~d} x$, where $C_{N}^{2}$ is the strength of the electron-density fluctuations ${ }^{2}$ (Fig. 5). The results were parameterised by the screen velocity. We estimated the uncertainties in $D_{\text {scr }}$ and $\theta_{\mathrm{s}}^{F W H M}$ by Monte Carlo methods and the hatched regions in Fig. 5 show the $1 \sigma$ error for these quantities. In addition, the results given by the interpolation formula of Goodman (1997) were confirmed by numerically integrating the intensity covariance function for refractive scintillation (see Appendix A in Rickett et al. 2006). In the bottom panel of Fig. 5, we indicate an approximate lower limit to the source size, $\theta_{\mathrm{s}}^{F W H M} \geq 17 \mu \mathrm{as}$, given by the IC catastrophe limit and by assuming an upper limit of 50 for the Doppler factor (Lähteenmäki \& Valtaoja 1999; Cohen et al. 2007). As can be seen from Fig. 5, if the variability is due to ISS, we have either a rather nearby screen $\left(D_{\text {scr }} \lesssim 300 \mathrm{pc}\right)$ with $S M \gtrsim 0.5 \mathrm{~m}^{-20 / 3} \mathrm{pc}$ or $\theta_{\mathrm{s}}^{F W H M}$ so small that it would require Doppler factor exceeding 50 .

We can estimate $\theta_{\mathrm{s}}^{F W H M}$ independently by assuming that there is approximate equipartition between the energy densities of the magnetic field and the radiating electrons (Scott \& Readhead 1977). We assume the synchrotron peak frequency to be $15 \mathrm{GHz}$ and the corresponding peak flux density to be $1.5 \mathrm{Jy}$. This is based on the assumption that the scintillating component is the core of a Blandford \& Königl (1979) type jet and corresponds to the $\tau=1$ surface at our observing frequency. The resulting equipartition size is $\theta_{\mathrm{s}}^{F W H M} \sim 270 \cdot \delta^{-7 / 17} \mu$ as, where $\delta$ is the Doppler factor. If we again take $\delta \leq 50$, this results in $\theta_{\mathrm{s}}^{F W H M} \gtrsim 55 \mu \mathrm{as}, S M \gtrsim 4 \mathrm{~m}^{-20 / 3} \mathrm{pc}$, and $D_{\text {scr }} \lesssim 100 \mathrm{pc}$ for screen velocities between 10 and $50 \mathrm{~km} \mathrm{~s}^{-1}$. Since the Galactic electron distribution model by Cordes \& Lazio (2002) predicts a scattering measure of only $0.1 \mathrm{~m}^{-20 / 3} \mathrm{pc}$ for the line-of-sight towards $1156+295$, our results indicate a nearby, localised region of highly turbulent ionised gas in that direction.

The frequency-dependence of $m$ is slightly surprising: one would not expect $m$ to increase from $5.8 \%$ at $5 \mathrm{GHz}$ (Lovell et al. 2003) to $13 \%$ at $15 \mathrm{GHz}$, unless the scattering is strong

\footnotetext{
${ }^{2}$ Note that $\theta_{\mathrm{s}}$ in Goodman (1997) is approximately $\theta_{\mathrm{s}}^{F W H M} / 2.35$.
}

and the source is smaller than the scattering angle. In that case the scintillating component would contain only about $30-50 \%$ of the total flux density of the core, because otherwise the observed $m$ would be significantly higher. Another possibility is that $1156+295$ was in a more compact stage during our observation than it was 5 years earlier. Simultaneous multi-frequency measurements of $m$ are required to clarify this.

It is also possible that the observed variations are not due to standard ISS, but instead correspond to an isolated extreme scattering event (ESE; Fiedler et al. 1987). Unfortunately, our short, single-frequency observation does not provide us with sufficient information to distinguish between these two cases. However, the shape of the flux density curve resembles an ESE with two maxima symmetrically surrounding a deep minimum. Although "classical" ESEs have durations of between weeks and months, Cimò et al. (2002) reported an event in 0954+658 with a duration of less than 2 days. The extremely short timescale of our event would, in the case of an ESE, imply a cloud size $<0.01 \mathrm{AU}$.

Acknowledgements. We thank Lars Fuhrmann, David Jauncey, Richard Porcas, Eduardo Ros and the anonymous referee for their careful reading of the manuscript and their constructive comments. We also thank Thomas Krichbaum, Matthew Lister and Sarma Kuchibhotla for useful discussions. Both authors are research fellows of the Alexander von Humboldt Foundation. T.S. was also partially supported by the Max-Planck-Gesellschaft and by the Academy of Finland grant 120516. The National Radio Astronomy Observatory is a facility of the National Science Foundation operated under cooperative agreement by Associated Universities, Inc.

\section{References}

Begelman, M. C., Rees, M. J., \& Sikora, M. 1994, ApJ, 429, L57 Bignall, H. E., Jauncey, D. L., Lovell, J. E. J., et al. 2003, ApJ, 585, 653 Bignall, H. E., Macquart, J.-P., Jauncey, D. L., et al. 2006, ApJ, 652, 1050 Blandford, R. D., \& Königl, A. 1979, ApJ, 232, 34 Burbidge, E. M. 1968, ApJ, 154, L109

Cimò, G., Beckert, T., Krichbaum, T. P., et al. 2002, PASA, 19, 10 Cohen, M. H., Lister, M. L., Homan, D. C., et al. 2007, ApJ, 658, 232 Cordes, J. M., \& Lazio, T. J. W. 2002 [arXiv: astro-ph/0207156] Dennett-Thorpe, J., \& de Bruyn, A. G. 2002, Nature, 415, 57 Dennett-Thorpe, J., \& de Bruyn, A. G. 2003, A\&A, 404, 113 Fiedler, R. L., Dennison, B., Johnston, K. J., et al. 1987, Nature, 326, 675 Fuhrmann, L., Krichbaum, T. P., Cimò, G., et al. 2002, PASA, 19, 64 Gabányi, K. É., Marchili, N., Krichbaum, T. P., et al. 2007, A\&A, 470, 83 Goodman, J. 1997, New Astron., 2, 449

Heeschen, D. S., Krichbaum, T., Schalinski, C. J., et al. 1987, AJ, 94, 1493 Hong, X. Y., Jiang, D. R., Gurvits, L. I., et al. 2004, A\&A, 417, 887 Jauncey, D. L., \& Macquart, J.-P. 2001, A\&A, 370, L9

Jauncey, D. L., Kedziora-Chudczer, L. L., Lovell, J. E. J., et al. 2000, in Astrophysical Phenomena Revealed by Space VLBI, ed. H. Hirabayashi, P. G. Edwards, \& D. W. Murphy, 147

Jauncey, D. L., Johnston, H. M., Bignall, H. E., et al. 2003, Ap\&SS, 288, 63 Jorstad, S. G., Marscher, A. P., Mattox, J. R., et al. 2001, ApJS, 134, 181

Kedziora-Chudczer, L. L., Jauncey, D. L., et al. 2001, MNRAS, 325, 1411 Kellermann, K. I., \& Pauliny-Toth, I. I. K. 1969, ApJ, 155, L71

Kellermann, K. I., Lister, M. L., Homan, D. C., et al. 2004, ApJ, 609, 539

Kovalev, Y. Y., Kovalev, Y. A., Nizhelsky, N. A., et al. 2002, PASA, 19, 83 Kovalev, Y. Y., Kellermann, K. I., Lister, M. L., et al. 2005, AJ, 130, 2473 Kraus, A., Krichbaum, T. P., Wegner, R., et al. 2003, A\&A, 401, 161

Lähteenmäki, A., \& Valtaoja, E. 1999, ApJ, 521, 493

Lister, M. L., \& Homan, D. C. 2005, AJ, 130, 1389

Lovell, J. E. J., Jauncey, D. L., Bignall, H. E., et al. 2003, AJ, 126, 1699

Piner, B. G., \& Kingham, K. A. 1997, ApJ, 485, L61

Raiteri, C. M., Ghisellini, G., Villata, M., et al. 1998, A\&AS, 127, 445

Rickett, B. J. 1990, ARA\&A, 28, 561

Rickett, B. J., Witzel, A., Kraus, A., et al. 2001, ApJ, 550, L11

Rickett, B. J., Lazio, T. J. W., \& Ghico, F. D. 2006, ApJS, 165, 439

Scott, M. A., \& Readhead, A. C. S. 1977, MNRAS, 180, 539

Sreekumar, P., Bertsch, D. L., Dingus, B. L., et al. 1996, ApJ, 464, 628

Wagner, S. J., \& Witzel, A. 1995, ARA\&A, 33, 163

Wills, B. J., Pollock, J. T., Aller, H. D., et al. 1983, ApJ, 274, 62

Witzel, A., Heeschen, D. S., Schalinski, C., \& Krichbaum, T. 1986, Mitteilungen der Astronomischen Gesellschaft Hamburg, 65, 239 